\title{
Game Theoretic Approach for Power Control using Error Control Coding in Wireless Sensor Networks
}

\author{
R.Valli \\ Department of ECE \\ Pondicherry Engineering College
}

\author{
A.Sharmila \\ Department of ECE \\ Pondicherry Engineering College
}

\author{
P.Dananjayan \\ Department of ECE \\ Pondicherry Engineering College
}

\begin{abstract}
Recent advances in electronic and computer technologies have paved the way for explosion of wireless sensor networks. Sensor network usually consists of a large number of ultra small autonomous devices. The resource constraint nature of these ultra small devices poses an immediate need for resource management. A fundamental component of resource management is transmitter power control and an efficient power control technique is essential to support system quality and efficiency in wireless sensor networks. The data transmitted from the sensor nodes is highly susceptible to error in a wireless environment which increases the transmit power. Error control coding (ECC) schemes can improve the system performance and has an impact on energy consumption as node energy is sturdily influenced by the modulation and error correction coding used. This paper proposes a power control solution for wireless sensor network (WSN) considering ECC in the analytical setting of a game theoretic approach. The game is formulated as a utility maximizing distributed power control game while considering the cost function and the existence of Nash equilibrium is studied. With the help of this equilibrium a distributed power control algorithm is devised. From the analysis it is evident that the system is power stable only if the nodes comply with certain transmit power. The utility of nodes employing ECC and without ECC is compared; the results show that the proposed algorithm employing ECC achieves the best response for the sensor nodes by consuming less power.
\end{abstract}

\section{General Terms}

Wireless sensor network, Power control, Game theory

\section{Keywords}

Reed Solomon codes, Nash Equilibrium, Utility

\section{INTRODUCTION}

The advancements in VLSI technology has led to the miniaturization of electronic devices and this coupled with wireless communication has proliferated the boom of tiny, low power, low cost sensor nodes. Energy consumption is a major constraint in wireless sensor network (WSN) as it determines the lifetime of a sensor and that of a sensor network. Battery capacity is limited and it is usually impossible to replace them. Any operation performed on a sensor consumes energy, involving discharge of battery power. Hence efficient use of resources grows as an important issue in wireless sensor networks. Among various resource management methods power control in WSN is significant to overcome unnecessary interference and to save the battery life of the sensors.

The design of dexterous power control algorithms for wireless sensor networks is decisive to reduce energy consumption to a level appropriate for many applications [1]. This is for instance the case in the disaster relief scenario, where wireless sensor nodes are used to provide real-time information on the physical conditions in a military environment. In such a circumstance, where recharging is typically not possible, radio power control is vital in order to increase the network lifetime. Transmission power is responsible for up to $70 \%$ of the total energy consumption for off-the-shelf sensor nodes, [2]. Keeping the transmit power under control is moreover favourable to trim down the packet collision probability, which if not leads to more retransmitted packets wasting even more energy.

Furthermore, in wireless environments, multipath fading strongly impacts the communication in WSN. Multipath fading increases the possibility of signal cancellation which leads to higher packet loss and therefore resulting in more power consumption. As sensors are used for high end applications such as radiation and nuclear threat detection systems, the data has to be reproduced with extremely low bit error rate (BER). To maintain the BER within a limit, either transmit signal power has to be increased or error control coding (ECC) can be used. ECC reduces the required transmitted signal energy because of its coding gain. Energy constraint transmission issue of WSN makes forward error correction (FEC) a popular error correction technique to be used in such networks. A system with FEC can provide a reliability using less power than a system without FEC [3]. Therefore proper error control coding can save the power required for communication of the information bits.

Many research efforts in recent years have focused on developing power saving schemes for wireless sensor networks. A considerable amount of research has been done on prolonging the wireless sensor network lifetime from the perspective of higher communication layers [4-6]. Researchers have explored the sensor node energy with different error control codes as well as different modulation schemes. Chouhan et al.[7] have proposed a framework for energy consumption based design space exploration. Using this framework, the authors have explored various ECCs and observed that using ECC saves energy as compared to uncoded data transmission. Howard et al. [8] have analyzed the distance at which an ECC becomes energy efficient for different environment and operating frequencies. For uncoded data transmission, effect of changing modulation constellation on the energy has been studied by Schurgers et al. [9]. Most of the pioneering research in the area of energy-constrained communication has focused on transmission schemes to minimize the transmission energy per bit. In [10], Verdu discusses some optimal strategies that minimize the energy per bit required for reliable transmission in the wideband regime. Among the most popular FEC's today Reed Solomon (RS) code is widely used and is considered to be the best choice for WSN having maximum energy efficiency in proper channel conditions or when relay nodes are sufficient in numbers i.e. greater than five[11]. 
Game theory has been effectively used in ad hoc and sensor networks for designing mechanisms to provoke desirable equilibria by contributing incentives to the forwarding nodes [12$15]$ and also grueling nodes for misbehaving. Chang and Tassiulas [16] investigated the energy efficiency problem in wireless sensor networks as the maximum network lifetime routing problem. They proposed to adjust the transmit power levels to just reach the intended next hop receiver such that the energy consumption rate per unit information transmission can be reduced. It has been well established that incorporating pricing schemes can stimulate a cooperative environment, which benefits both the network and the nodes.

In this paper, game theory is adopted for power control of wireless sensor networks and a non-cooperative game model is designed especially considering the benefits of ECC, where each node tries to maximize its net utility. Increasing the SINR of one node will impair the SINR of another node. The action of other nodes will affect the action and strategies of the considered node making this problem suitable for game theoretic analysis. Net utility is computed by considering the benefit received and the cost incurred for packet transmissions. Any information about the separate transmitting power level strategies taken by other nodes are not used which reduce the control signals greatly, thereby helping nodes in conserving energy.

The rest of the paper is organized as follows. Section II, deals with error control coding for WSN. The power control game is formulated and the existence of Nash Equilibrium is verified in section III. Simulation results are given and discussed in section IV. Finally, conclusions are drawn in Section V.

\section{ERROR CONTROL CODING FOR WSN}

Lack of efficient ECC in sensor networks contributes their weak bit error rate performance in wireless environments where high levels of noise and interference are unavoidable. For recovering the erroneous packets, three fundamental schemes are Forward Error Correction (FEC), Automatic Repeat Request (ARQ) and Hybrid ARQ (HARQ). ARQ is very simple but it involves additional retransmission energy cost and area overhead. HARQ combines ARQ and FEC. It consumes a lot of energy and is restricted to some specific applications. The main advantage with FEC is that there are no delays in message flows. Energy constrained transmission issue of WSN makes FEC a popular technique to be used in such networks rather than ARQ and HARQ. Reed Solomon codes are long time industry standard codes for WSN.

For RS code,

$k:$ Number of information symbols.

$n:$ Length of the code word.

$T$ : Maximum number of the errors that a code can correct.

where $k=n-2 T$.

RS code is represented as $R S\left(n, k, d_{\text {min }}\right)$, where $\mathrm{d}_{\text {min }}$ represents the minimum distance of the code and $\mathrm{d}_{\min }=2 \mathrm{~T}+1$.

For FSK SNR coded $_{\text {is expressed as }}$

$S I N R_{\text {coded }} \leq \frac{n}{n-2 T} \frac{2}{b}\left(\operatorname{erfc}^{-1}\left(\frac{2 p_{s}}{2^{b}-1}\right)\right)^{2}$

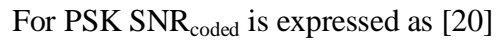

$$
S I N R_{\text {coded }}=\left\{\begin{array}{l}
\frac{n}{n-2 T}\left(e r f c^{-1}\left(2 p_{s}\right)\right)^{2}, b=1 \\
\frac{n}{n-2 T} \frac{\left(e r f c^{-1}\left(2 p_{s}\right)\right)^{2}}{b\left(\sin \frac{\pi}{2^{b}}\right)}, \text { otherwise }
\end{array}\right.
$$

where $p_{s}$ is the channel symbol error probability corresponding to the desired coded symbol error probability $\left(\mathrm{p}_{\mathrm{es}}\right)$ or coded bit error probability $\left(\mathrm{p}_{\mathrm{eb}}\right)$.

\section{GAME THEORETIC APPROACH}

Game theory is a mathematical study of conflict and cooperation between rational and intelligent entities. It is a tool for analyzing the interaction of decision makers. Game theory provides a suite of modeling tools for analyzing interactive scenarios and strives to predict their possible outcomes. Formally, a game has the following three components:

Player set $\mathrm{N}: \mathrm{N}=\{1,2, \ldots \mathrm{N}\}$ where $\mathrm{n}$ is the number of players in the game.

Action set $A: A=\times_{i=1}^{n} A_{i}$ is the space of all action vectors, where each component, $a_{i}$ of the vector $a \in A$ belongs to the set $\mathrm{A}_{\mathrm{i}}$, the set of actions of player $i$. Often the action profile is denoted by $a=\left(a_{i}, a_{-i}\right)$ where $a_{i}$ is player i's action and $a_{-i}$ denotes the actions of the other n-1 players. Similarly, $A_{-i}=\times_{j \neq i} A_{j}$ is used to denote the set of action profiles for all players except i.

Utility u: For each player $\mathrm{i} \in \mathrm{N}$, utility function $u_{i}: A \rightarrow R$ captures the player's preferences over outcomes determined by the action profiles. $\boldsymbol{u}=\left(\boldsymbol{u}_{1}, \boldsymbol{u}_{2}, \ldots . u_{n}\right): A \rightarrow R^{n}$ denotes the vector of such utility functions.

One of the goals of game theory is to predict the likely outcome of a game. The Nash Equilibrium (NE) is perhaps the most wellstudied and generalized solution concept in game theory. Most other equilibrium concepts are, in one form or another, refinements, extensions or derivatives of the NE concept. If for all $\mathrm{i} \in \mathrm{N}$, and $p_{i} \in P_{i}, u_{i} p_{i}, p_{-i} \geq u_{i} p_{i}^{*}, p_{-i}$, then power vector $P$ is the Nash Equilibrium of the power control game. An $\mathrm{NE}$ is a stable point from which no rational player has any incentive to unilaterally alter his action. Nash Equilibrium point is a balance point in which no participating nodes will deviate from in the Game. Therefore, in some sense, an NE is a consistent predictor of the outcome of a game.

\subsection{Game Theoretic Approach for Power Control}

The proposed model consist of $\mathrm{N}$ nodes in the network, $\mathrm{d}_{\mathrm{i}}$ is the distance between node $\mathrm{i}$ and sink node, $\mathrm{p}_{\mathrm{i}}$ and $\gamma_{\mathrm{i}}$ are the transmitting power and receive signal interference noise of node $i$, hi denotes the link gain from node $\mathrm{i}$ to sink node. The nodes in the network play repeated game. The information from earlier rounds are used to work out strategies in upcoming rounds. A particular node with potentially as many as $\mathrm{N}$ neighbours within the interference range is considered. The problem of communication between neighbouring nodes in a network that consists of sensor 
nodes scattered randomly over an area is considered. The sensor nodes have limited energy, buffer space, and other resources, hence contention-based protocols may not be a suitable option. Here, as an alternative, code division multiplexing, is used where distinct codes can be allocated to different nodes with possible code reuse between spatially separated nodes. The receiving and interference ranges for each sensor node depend on the transmission power of the sender and the other sensor nodes in vicinity. The receiving distance, is defined as the maximum distance from which a receiving node can correctly recover a transmitted signal.

All the nodes can transmit with any power level to make its transmission successful. Also, the nodes have no information if the other nodes are transmitting, hence leading to an incomplete information situation [17]. If the nodes transmit with an arbitrary high power level, it will increase the interference level of the other nodes. The neighbouring nodes in turn will transmit at higher power to overcome the effect of high interference. Soon, this will lead to a non cooperative situation. To control this non cooperative behavior, an equilibrium game strategy which will impose constraints on the nodes to act in cooperative manner even in a non cooperative network is devised.

The existence of some strategy sets $\mathrm{P}_{1}, \mathrm{P}_{2}, \ldots, \mathrm{P}_{\mathrm{N}+1}$ for the nodes $1,2, \ldots,(\mathrm{N}+1)$ is assumed. These sets consist of all possible power levels ranging from the minimum transmit power $p_{\min }$ to maximum transmit power $p_{\max }$.

In this game, if node 1 chooses its power level $p_{1} \in P_{1}$, and node 2 chooses its power level $p_{2} \in P_{2}$, and so on, then,

$p_{1} \in P_{1}$

(3)

This vector of individual strategies is called a strategy profile.

The set of all such strategy profiles is called the space of strategy profiles $P^{\prime}$. The game is played by having all the nodes concurrently pick their individual strategies. This set of choices results in some strategy profile $p \in P^{\prime}$, and is called as the outcome of the game. Each node has a set of preferences over these outcomes $p \in P^{\prime}$.

At the end of an action, each node $i \epsilon I$ receives a utility value

$u_{i} p=u_{i} p_{i}, p_{-i}$

$p_{-i}$ is the strategy profile of all the nodes but for the ith node.

The utility to any one node depends on the entire strategy profile. During every game, the node decides whether to transmit or not, rise or lower its power level, and chooses a power level if it decides to transmit. The $i^{\text {th }}$ node has control over its own power level $p_{i}$ only, and the utility if a node is transmitting by expending $p_{j}$ is given as

$u_{i} \quad p_{i}, p_{-i}=\frac{b R}{f p_{i}} f \gamma_{j}$

$b$ is the number of information bits in a packet of size $F$ bits.

$R$ is the transmission rate in bits/sec using strategy $p_{i}$. $\left(f\left(\gamma_{j}\right)\right)$ is the efficiency function which increases with expected SINR of the receiving node.

The efficiency function, is defined as $f\left(\gamma_{j}\right)=\left(1-2 P_{e}\right)^{F}$ where $P_{e}$ is the bit error rate (BER) and $P_{e}$ is a function of SINR.

With a noncoherent FSK modulation scheme, $P_{e}=0.5 e^{\frac{-\gamma_{j}}{2}}$, and with a BPSK modulation scheme, $P_{e}=0.5 e^{\sqrt{\gamma_{j}}}$

where, $\gamma_{j}$ denotes the expected SINR of node $\mathrm{j}$. It is assumed that the utility value obtained by a node when it decides not to transmit is 0 .

The power expended is given by

$$
p_{j}=\frac{\gamma_{j} \sum_{i \neq j} h_{i} P_{i}+\sigma^{2}}{\left(\frac{W}{R}\right) h_{j}}
$$

where;

$\mathrm{W}$ is the available bandwidth

$\mathrm{h}$ is the path gain

Each sensor node tries to maximize its own utility by adjusting its own power optimally as given by utility function. The utility function from a sensor node's viewpoint considers the interference it gets from other nodes; however, it ignores the fact that this node imposes on itself in terms of drainage of energy. Pricing or regulating cost has been shown to be effective in regulating this externality, as it encourages the nodes to use resources more efficiently [17]. The cost component accounts for the energy consumed/drained by the sensor nodes with usage of resources (transmission power). If the strategy of the $\mathrm{i}^{\text {th }}$ node is to transmit at signal power $p_{i} \in P$, the cost incurred is a function

of $p_{i}$, which is denoted by $A p_{i} \cdot p_{i}$ is a random variable denoting transmitting signal power of $i^{\text {th }}$ node. A linear cost function is considered.

A $p_{i}=k \times p_{i}$

where $\mathrm{k}$ is a scaling factor.

\subsection{Power Control Algorithm}

Consider node $\mathrm{i}$ is transmitting data to node $\mathrm{j}$. Node $\mathrm{i}$ receives the sum of interference power $\sum_{j=1}^{N} h_{j \neq i} P_{j}$ from sink node. The pseudocode of the procedure is shown in the algorithm mentioned below.

Algorithm

Require: $P_{i}=\left\{p_{i, 1} \ldots \ldots \ldots, p_{i, \max }\right\}$

SINR

$\left\{\right.$ while node $_{i}$, receives data

do

Calculate $\mathrm{u}_{\mathrm{i}}\left(\mathrm{p}_{\mathrm{i}}, \mathrm{p}_{-\mathrm{i}}\right)$ \#using equation (5)

End while\}

\{while node ${ }_{i}$, transmitting data

do 
Calculate $\mathrm{p}_{\mathrm{i}}$ \# using equation (1), (2), (6)

If $\mathrm{p}_{\mathrm{i}}<\mathrm{p}_{\mathrm{t}}$ (Threshold power)

Transmit data using optimal power

Else

Not transmit

End while

\section{RESULTS}

The analysis of the proposed algorithm is carried out using MATLAB 7.0. The following simulation parameters were considered.

Table 1. Simulation parameters

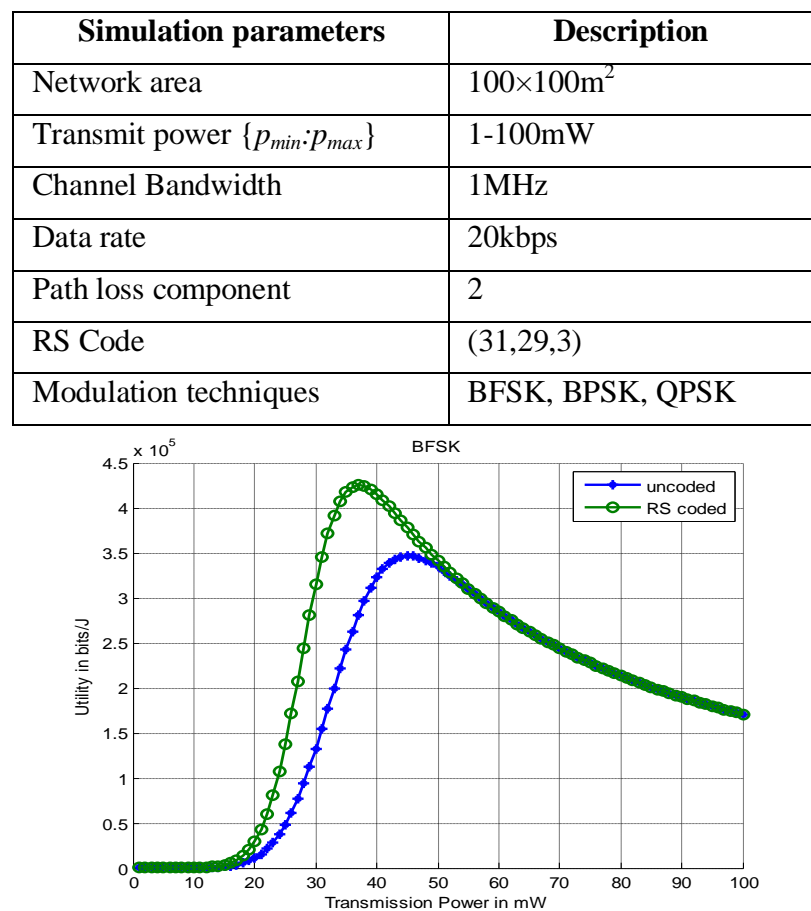

Figure 1 Utility for uncoded and RS coded schemes using BFSK modulation

Figure 1 shows the utility as the transmission power increases. There is an optimal value of $s_{i}$, beyond which the net utility only decreases. The figure serves as a guideline for calculating the desired transmitting power to maximize net utility. If node i's transmitting power is too low, then the node i's received power at the sink will be lower than the received powers of other nodes. This will cause the nodes SINR to be low and hurt its performance. This is reflected by the drop in the nodes utility function as $\mathrm{p}_{\mathrm{i}}$ approaches 0 . If the nodes transmit power is increased further and if it is too high, then it is wasting its battery power while having little impact on the bit error rate. This is reflected by the drop in the nodes utility function as $p_{i}$ approaches $\infty$. For BFSK without error control coding, a maximum utility of $3.5 \times 10^{5}$ bits/joule is achieved for a transmission power of $45 \mathrm{~mW}$, whereas with ECC an utility of $4.25 \times 10^{5}$ bits/joule is achieved for a transmission power of $36 \mathrm{~mW}$.

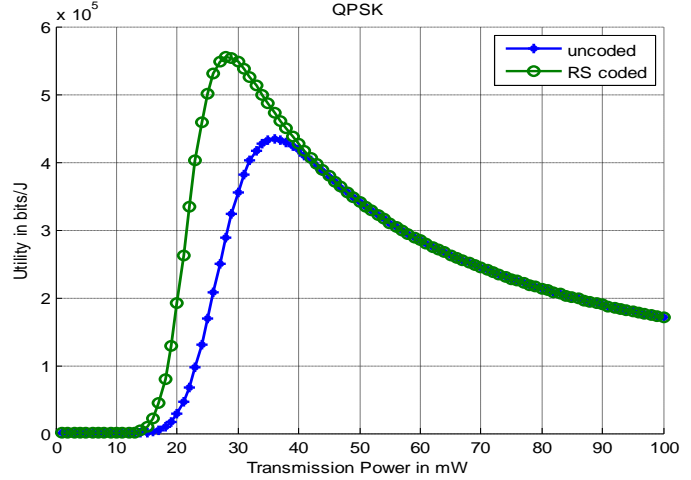

Figure 2 Utility for uncoded and RS coded schemes using QPSK modulation

Figure 2 shows that for QPSK without error control coding, a maximum utility of $4.3 \times 10^{5}$ bits/joule is achieved for a transmission power of $35 \mathrm{~mW}$, whereas with ECC an utility of $5.6 \times 10^{5}$ bits/joule is achieved for a transmission power of $29 \mathrm{~mW}$.

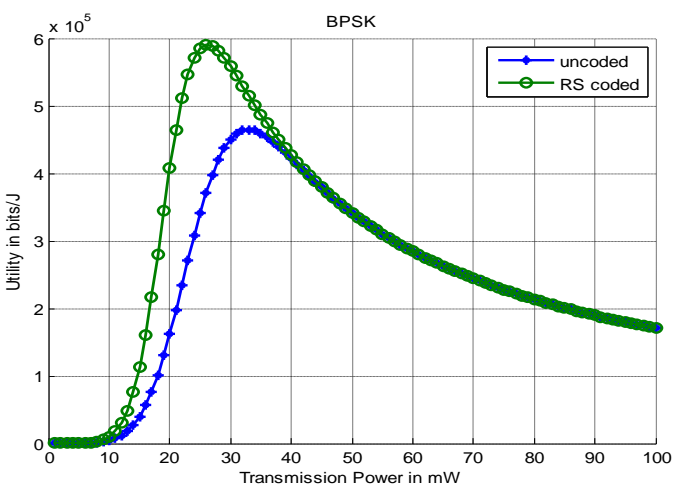

Figure 3 Utility for uncoded and RS coded schemes using BPSK modulation

Figure 3 shows that for BPSK without error control coding, a maximum utility of $4.5 \times 10^{5}$ bits/joule is achieved for a transmission power of $32 \mathrm{~mW}$, whereas with ECC an utility of $5.9 \times 10^{5}$ bits/joule is achieved for a transmission power of $26 \mathrm{~mW}$. The transmitting power lever of $26 \mathrm{~mW}$ gives the best response for the node. If a node unilaterally changes its strategy and does not transmit with this power level, then the node will not get its best response and will not be able to reach the Nash equilibrium.

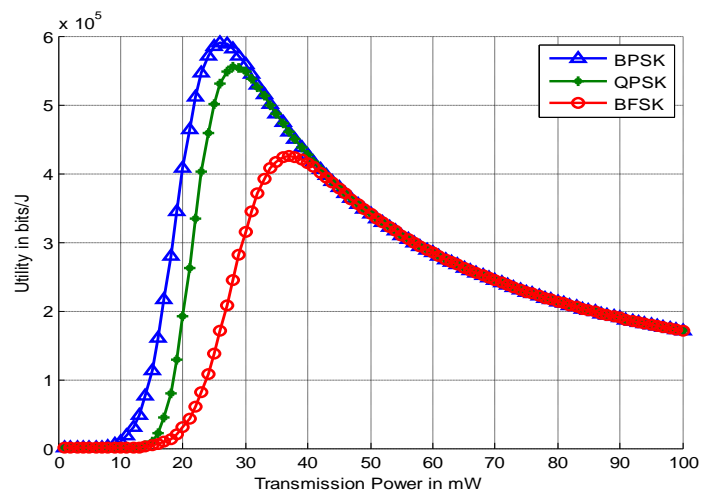

Figure 4 Comparison of utility for RS coded schemes 
From Figure 4 it is evident that BPSK provides an improvement of $7 \%$ in utility as compared with QPSK with the reduction in transmitted power by $11 \%$ and an improvement of $39 \%$ in utility as compared with BFSK with the reduction in transmitted power by $38 \%$.

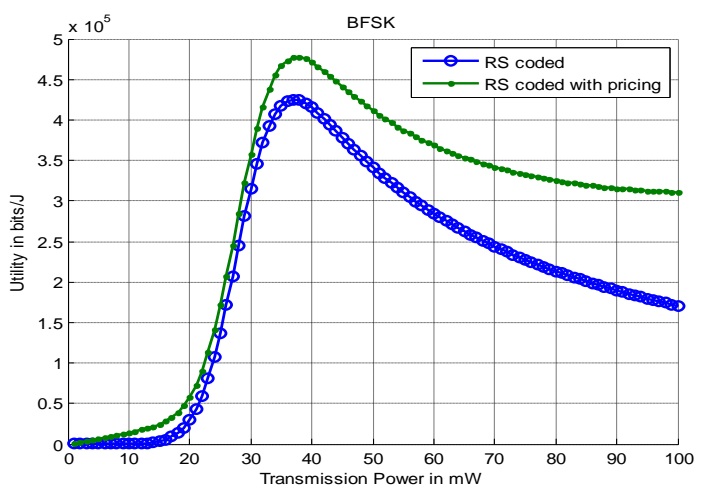

Figure 5 Utility for RS coded scheme with and without pricing using BFSK modulation

Fig. 5 shows that a maximum utility of $4.75 \times 10^{5}$ bits/joule is achieved for a transmission power of $29 \mathrm{~mW}$ when considering the cost function. A reward of $12 \%$ is received when compared to the scheme without pricing.

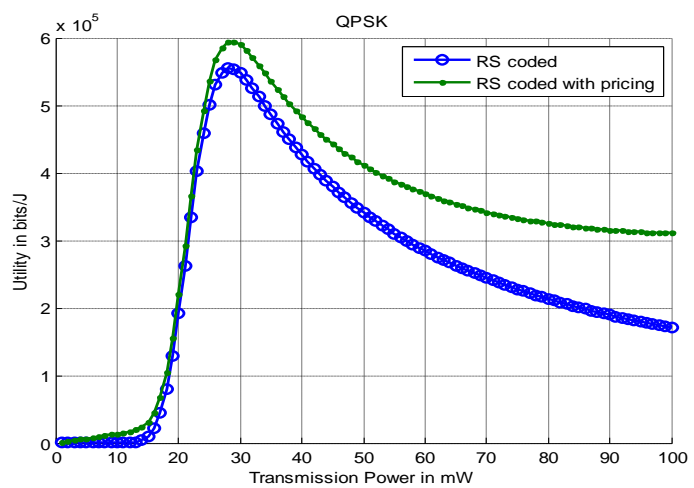

Figure 6 Utility for RS coded scheme with and without pricing using QPSK modulation

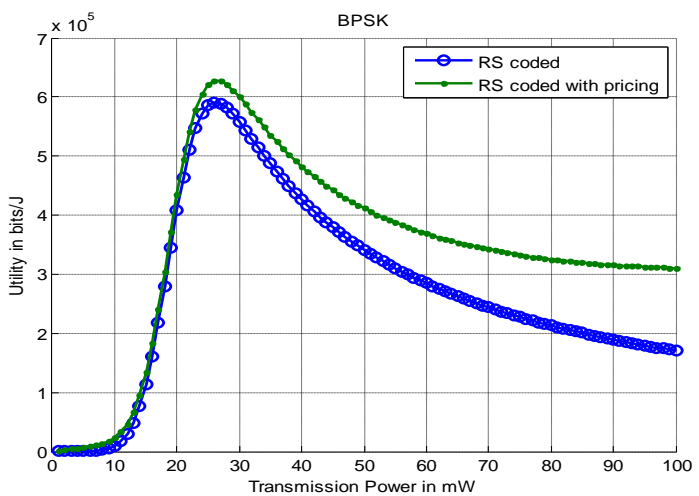

Figure 7 Utility for RS coded scheme with and without pricing using BPSK modulation

Figure 6 shows a maximum utility of $5.9 \times 10^{5}$ bits/joule is achieved for a transmission power of $29 \mathrm{~mW}$ when considering the cost function. A reward of $7 \%$ is received when compared to the scheme without pricing. A maximum utility of $6.3 \times 10^{5}$ bits/joule is achieved for a transmission power of $26 \mathrm{~mW}$ when considering the cost function is shown in Figure 7. This offers an incentive of nearly $7 \%$ when compared to the scheme without pricing.

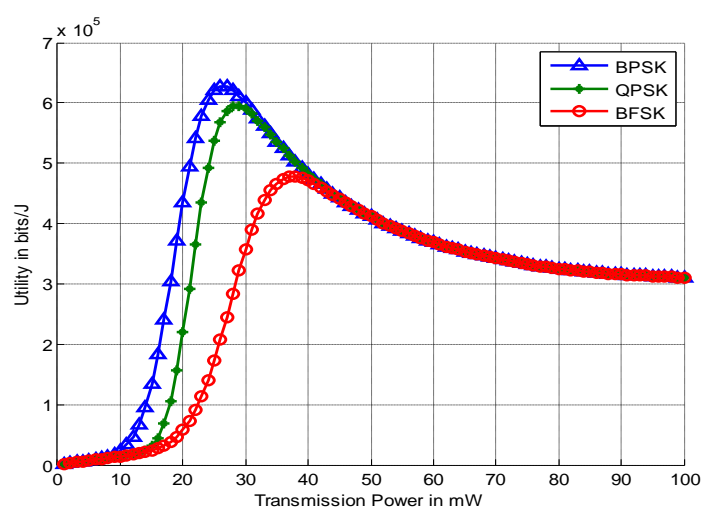

Figure 8 Comparison of utility for RS coded scheme with pricing

From Figure 8 it is intuitive that BPSK provides an improvement of $7 \%$ in utility is achieved as compared with QPSK with the reduction in transmitted power by $11 \%$ and an improvement of $39 \%$ in utility as compared with BFSK with the reduction in transmitted power by $38 \%$. Comparing BPSK with and without pricing an increase in utility of $7 \%$ is achieved for the same transmitting power $(26 \mathrm{~mW})$. Whereas comparing QPSK with and without pricing an increase in utility of $7 \%$ is achieved for the transmitting power of $29 \mathrm{~mW}$. BFSK with and without pricing scheme provides an increase in utility of $12 \%$ for the same transmitting power $(36 \mathrm{~mW})$.

\section{CONCLUSION}

Power control is an efficient way to conserving limited energy sensors. A game-theoretic approach with error control coding to solve the power control problem encountered in sensor networks is presented. The power control game is formulated and the existence and uniqueness of the Nash Equilibrium are studied for the proposed game model. The utility of nodes employing RS codes and without RS codes is compared. The outcome shows that the proposed algorithm employing RS codes achieves the best response for the sensor nodes by consuming less power. The power control algorithm proposed in the paper can reduce node transmitting power, save node energy and prolong network lifetime efficiently.

\section{REFERENCES}

[1] Fischione,C., Bonivento,A., Johansson, K. H., SangiovanniVincentelli, A., 2006. Cooperative diversity with disconnection constraints and sleep discipline for power control in wireless sensor networks. In Proceedings of IEEE Vehicular Technology Conference.

[2] Lymberopoulos,D., Lindsey,A., Savvides, 2006. Characterization of the radio signal strengh variability in 3-D IEEE 802.15.4 networks using monopole antennas. EWSN, ETH, Zurich,

[3] Shraddha Srivastava, Christian Spagnol, Emanuel Popovici, 2009. Ananlysis of a set of error correcting schemes in multihop wireless sensor networks. In Proceedings of IEEE PRIME 2009, Ireland. pp.1-4 
[4] Heinzelman,W., Chandrakasan,A., and Balakrishnan,H., 2002. An application-specific protocol architecture for wireless microsensor networks. IEEE Transactions on Wireless Communication.(Oct.2002), 660-670.

[5] Younis.O., and Fahmy,S., 2004. Distributed Clustering in adhoc sensor networks: A hybrid energy efficient approach. In Proceedings of $23^{\text {rd }}$ Annual Joint Conference of the IEEE Computer and Communications in Societies(INFOCOM).

[6] Schurgers,C., Tsiatsis,V., Ganeriwal,S., and Srivastava,M., 2002. Optimizing sensor networks in the energy-latencydensity design space. IEEE Transactions on Mobile computing (Jan. 2002) 70-80.

[7] Chouhan,S., Bose,R., and Balakrishnan,M., (2009). A framework for energy consumption based design space exploration for wireless sensor nodes. IEEE Transaction on Computer-Aided Design Integrated Circuits System. July 2009, 1017-1024.

[8] Howard,S.L., Schlegel,C., and Iniewski,K., (2006). Error control coding in low power wireless sensor networks: when is ECC energy efficient? EURASIP Journal on Wireless Communications and Networking. 1-14.

[9] Schurgers,C., Aberthrone,O., and Shrivastava,M.B., (2001). Modulation scaling for energy aware communication systems. In Proceedings of International Symposium on Low Power Electronic Design. 96-99.

[10] Verdu,S., (2002). Spectral efficiency in the wideband regime. IEEE Transaction on Information Theory, (June 2002) 13191343.

[11] Zeinab Hajjarian Kashani and Mohsen Shiva, 2006. Channel Coding in Multihop Wireless Sensor Network. In Proceedings of International Conference on ITS Telecommunications.

[12] Buchegger,S., and Le Boudec,J., (2002). Performance Analysis of the CONFIDANT Protocol. In Proceedings of Third ACM International Symposium on Mobile AdHoc Networking \& Computing, 226-232.

[13] Buttyan,L., and Hubaux,J.P., (2001). Nuglets: A Virtual Currency to Stimulate Cooperation in Selforganized Mobile Ad-Hoc Networks. Technical Report DSC/2001/001, Swiss Federal Institute Of Technology, (Jan. 2001).

[14] Wang,W., Chatterjee,M., and Kwiat,K., (2008). Enforcing Cooperation in Ad Hoc Networks with Unreliable Channel.
In Proceedings of Fifth IEEE International Conference on Mobile Ad-Hoc and Sensor Systems (MASS), 456-462.

[15] Srinivasan,V., Nuggehalli,P., Chiasserini,C., and Rao,R., (2003). Cooperation in Wireless Ad Hoc Networks. In Proceedings of IEEE INFOCOM, (Apr.2003) 808-817.

[16]Chang,J., and Tassiulas,L.,(2004) Maximum Lifetime Routing in Wireless Sensor Networks. IEEE/ACM Transaction on Networking, (Aug. 2004), 609-619.

[17] Shamik Sengupta, Mainak Chatterjee, and Kevin A. Kwiat,(2010). A Game Theoretic Framework for Power Control in Wireless Sensor Networks. IEEE Transactions on Computers, (Feb. 2010), 231-242

\section{AUTHORS PROFILE}

R.Valli received B.E degree in Electronics and Communication Engineering from Madras University, Chennai in 1996 and M.Tech degree in Electronics and Communication Engineering from Pondicherry Engineering College, Pondicherry in 2005. She is pursuing her Ph.D. in the Department of Electronics and Communication Engineering, Pondicherry Engineering College, Pondicherry. Her research interests include computer networks, wireless adhoc and sensor network.

A.Sharmila received her B.Tech degree in Electronics and Instrumentation Engineering from Pondicherry Engineering College, Pondicherry in 2009 and is pursuing her M.Tech degree in Wireless Communication from the same institution. Her area of interests includes digital and wireless communication.

P. Dananjayan received Bachelor of Science from University of Madras in 1979, Bachelor of Technology in 1982 and Master of Engineering in 1984 from the Madras Institute of Technology, Chennai and Ph.D. degree from Anna University, Chennai in 1998. He is working as Professor in the Department of Electronics and Communication Engineering, Pondicherry Engineering College, Pondicherry, India. He has been as visiting professor to AIT, Bangkok. He has to his credit more than 75 publications in National and International Journals. He has presented more than 160 papers in National and International Conferences. He has guided $9 \mathrm{Ph} . \mathrm{D}$ candidates and is currently guiding $6 \mathrm{Ph} . \mathrm{D}$ students. His research interests include spread spectrum techniques, wireless communication, wireless adhoc and sensor networks. 